\title{
Dynamics analysis on a class of delayed neural networks involving inertial terms
}

\author{
Jian Zhang ${ }^{1 *}$ and Chuangxia Huang ${ }^{1}$
}

\author{
"Correspondence: \\ jianzhangmath@126.com \\ ${ }^{1}$ School of Mathematics and \\ Statistics, Hunan Provincial Key \\ Laboratory of Mathematical \\ Modeling and Analysis in \\ Engineering, Changsha University of \\ Science and Technology, Changsha, \\ P.R. China
}

\begin{abstract}
This paper explores a class of unbounded distributed delayed inertial neural networks. By introducing some new differential inequality analysis and abandoning the traditional order reduction technique, some new assertions are derived to verify the global exponential stability of the addressed networks, which improve and generalize some recently published articles. Finally, two cases of numerical examples and simulations are given to illustrate these analytical conclusions.
\end{abstract}

Keywords: Distributed delayed inertial neural networks; Non-reduced order technique; Exponential stability

\section{Introduction}

In dynamic systems of neural networks, the existence of time delays is inevitable and is always a source of instability, chaos, and oscillation of the network system. In particular, on account of the occurrence of a lot of parallel routes with a series of different axon sizes and lengths, it is desired to account for the dynamical behaviors of neural networks by involving unbounded and continuously distributed delays. Meanwhile, there have existed many papers dealing with the dynamics studies of distributed delayed neural networks, which include stability analysis [1-4], almost periodic oscillation [5-9], and anti-periodic oscillation [10-12].

On the other hand, during the last two decades, by using reduced order technique, the famous inertial neural networks with constant delays and bounded time-varying delays have been widely investigated by many authors [13-37]. To reveal the rate of convergence, the exponential stability of inertial neural networks models with bounded delays has been extensively researched in [15-21, 29, 37] by changing the addressed models into the first order systems with some variable substitutions. Most recently, the authors in $[36,37]$ pointed out that the above transformation will generate new parameters and increase the dimension of the addressed systems, which is difficult to be achieved in practical problems. In addition, abandoning the traditional reduced-order technique, the authors of [36, 37] gained some new criteria to verify the synchronization and stability of the constant delayed inertial neural network involving a new Lyapunov functional, which are complementary to some known ones in $[13,35]$. Unfortunately, there is no published paper touching upon the exponential stability on continuously distributed delayed inertial neural network models.

(c) The Author(s) 2020. This article is licensed under a Creative Commons Attribution 4.0 International License, which permits use, sharing, adaptation, distribution and reproduction in any medium or format, as long as you give appropriate credit to the original author(s) and the source, provide a link to the Creative Commons licence, and indicate if changes were made. The images or other third party material in this article are included in the article's Creative Commons licence, unless indicated otherwise in a credit line to the material. If material is not included in the article's Creative Commons licence and your intended use is not permitted by statutory regulation or exceeds the permitted use, you will need to obtain permission directly from the copyright holder. To view a copy of this licence, visit http://creativecommons.org/licenses/by/4.0/. 
Based on the above arguments, we shall use the non-reduced order technique to establish the global exponential stability for the following unbounded continuously distributed delayed inertial neural networks:

$$
\begin{aligned}
x_{i}^{\prime \prime}(t)= & -a_{i} x_{i}^{\prime}(t)-b_{i} x_{i}(t)+\sum_{j=1}^{n} c_{i j} P_{j}\left(x_{j}(t)\right)+\sum_{j=1}^{n} d_{i j} \int_{0}^{+\infty} K_{i j}(u) \\
& \times Q_{j}\left(x_{j}(t-u)\right) d u+J_{i}(t), \quad t \geq 0, i \in D:=\{1,2, \ldots, n\},
\end{aligned}
$$

where $x_{i}^{\prime \prime}(t)$ is labeled as an inertial term of the state vector $x(t)=\left(x_{1}(t), x_{2}(t), \ldots, x_{n}(t)\right)$ in (1.1), the constants $a_{i}>0, b_{i}>0, c_{i j}$, and $d_{i j}$ are the connection weight parameters, the bounded external input $J_{i} \in C(\mathbb{R}, \mathbb{R})$ is continuous on $\mathbb{R}$, the delay kernel $K_{i j} \in$ $C([0,+\infty), \mathbb{R}), P_{j}$ and $Q_{j}$ are the activation functions with Lipschitz constants $L_{j}^{P}$ and $L_{j}^{Q}$ obeying

$$
\left|P_{j}(u)-P_{j}(v)\right| \leq L_{j}^{P}|u-v|, \quad\left|Q_{j}(u)-Q_{j}(v)\right| \leq L_{j}^{Q}|u-v|, \quad \forall u, v \in \mathbb{R}, j \in D .
$$

In (1.1), we define

$$
x_{i}(s)=\varphi_{i}(s), \quad x_{i}^{\prime}(s)=\psi_{i}(s), \quad-\infty<s \leq 0, i \in D,
$$

where $\varphi_{i}$ and $\psi_{i}$ are bounded and continuous initial values on $(-\infty, 0]$.

For the purpose of obtaining our main results in this paper, we presume the assumptions as follows:

$\left(T_{1}\right)$ For $i, j \in D$, there is $\kappa \in(0,+\infty)$ such that $\left|K_{i j}(t)\right| e^{\kappa t}$ is integrable on $[0,+\infty)$.

$\left(T_{2}\right)$ For $i \in D$, constants $\beta_{i}>0$ and $\alpha_{i} \geq 0, \gamma_{i} \geq 0$ can be present to agree with that

$$
E_{i}<0, \quad 4 E_{i} F_{i}>H_{i}^{2},
$$

where

$$
\left\{\begin{aligned}
E_{i}= & \alpha_{i} \gamma_{i}-a_{i} \alpha_{i}^{2}+\frac{1}{2} \sum_{j=1}^{n}\left(\left|c_{i j}\right| L_{j}^{P}+\left|d_{i j}\right| L_{j}^{Q} \int_{0}^{+\infty}\left|K_{i j}(u)\right| d u\right) \alpha_{i}^{2}, \\
F_{i}= & -b_{i} \alpha_{i} \gamma_{i}+\frac{1}{2} \sum_{j=1}^{n} \alpha_{j}^{2}\left(\left|c_{j i}\right| L_{i}^{P}+\left|d_{j i}\right| L_{i}^{Q} \int_{0}^{+\infty}\left|K_{j i}(u)\right| d u\right) \\
& +\frac{1}{2} \sum_{j=1}^{n}\left(\left|c_{j i}\right| L_{i}^{P}+\left|d_{j i}\right| L_{i}^{Q} \int_{0}^{+\infty}\left|K_{j i}(u)\right| d u\right)\left|\alpha_{j} \gamma_{j}\right| \\
& +\frac{1}{2} \sum_{j=1}^{n}\left(\left|c_{i j}\right| L_{j}^{P}+\left|d_{i j}\right| L_{j}^{Q} \int_{0}^{+\infty}\left|K_{i j}(u)\right| d u\right)\left|\alpha_{i} \gamma_{i}\right|, \\
H_{i}= & \beta_{i}+\gamma_{i}^{2}-\alpha_{i}^{2} b_{i}-a_{i} \gamma_{i} \alpha_{i} .
\end{aligned}\right.
$$

Remark 1.1 Since (1.1) can be converted into the first order functional differential equations, from (1.2), $\left(T_{1}\right)$, and the basic theory on infinite delayed differential equation in [38], one can show the existence and uniqueness of every solution of (1.1) and $(1.3)$ on $[0,+\infty)$.

Remark 1.2 Assumption ( $\left.T_{2}\right)$ means that $a_{i}$ and $b_{i}$ are large enough and satisfy the above matrix inequalities which are adopted to guarantee the stability of system (1.1). Clearly, the above matrix inequalities are weaker than those used in [15-21], where $\left|a_{i}-b_{i}\right|$ is assumed to be small enough. 
The main principle of this article is by employing differential inequality analysis to establish the global exponential stability of the addressed networks. To do that, our contributions are based on four aspects: (1) Propose a general inertial neural that has unbounded continuously distributed delays and is more general than the ones considered in [15-37]. (2) Establish sufficient conditions to ensure the global exponential stability of system (1.1). This is the first time to derive such a result for this type of unbounded continuously distributed delay inertial neural networks. (3) The results obtained in this article are original and complete those obtained previously in [17-21, 36, 37]. (4) The theoretical results play an important role in the design of the electrical implementation of the unbounded delayed inertial neural networks and in the processing of the transmission of its signals.

In the rest of this paper, Sect. 2 gives the global exponential convergence of all solutions with their derivatives of networks (1.1) under conditions (1.2), $\left(T_{1}\right)$, and $\left(T_{2}\right)$. Section 3 shows numerical figures. Conclusions are drawn in the last section.

\section{Global exponential stability}

Theorem 2.1 Under (1.2), ( $\left.T_{1}\right)$, and $\left(T_{2}\right)$, system (1.1) is globally exponentially stable. More precisely, label $x(t)=\left(x_{1}(t), x_{2}(t), \ldots, x_{n}(t)\right)$ and $y(t)=\left(y_{1}(t), y_{2}(t), \ldots, y_{n}(t)\right)$ as two solutions of system (1.1) satisfying

$$
x_{i}(s)=\varphi_{i}^{x}(s), \quad x_{i}^{\prime}(s)=\psi_{i}^{x}(s), \quad y_{i}(s)=\varphi_{i}^{y}(s), \quad y_{i}^{\prime}(s)=\psi_{i}^{y}(s), \quad i \in D,
$$

where $\varphi_{i}^{x}, \psi_{i}^{x}, \varphi_{i}^{y}$, and $\psi_{i}^{y}$ are bounded and continuous on $(-\infty, 0]$. Then, one can take two positive constants $\lambda$ and $\Lambda=\Lambda_{\varphi^{x}, \psi^{x}, \varphi^{y}, \psi^{y}}$ such that

$$
\left|x_{i}(t)-y_{i}(t)\right| \leq \Lambda e^{-\lambda t}, \quad\left|x_{i}^{\prime}(t)-y_{i}^{\prime}(t)\right| \leq \Lambda e^{-\lambda t}, \quad \forall t \geq 0, i \in D
$$

Remark 2.1 In Theorem 2.1, $x(t)$ and $x^{\prime}(t)$ are exponentially convergent to $y(t)$ and $y^{\prime}(t)$, respectively. This suggests that the stability on system (1.1) is in accordance with the exponential stability definition adopted in [15-21, 29].

Proof of Theorem 2.1 Label $w_{i}(t)=y_{i}(t)-x_{i}(t)(i \in D)$, then, for $i \in D$,

$$
\begin{aligned}
w_{i}^{\prime \prime}(t)= & -a_{i} w_{i}^{\prime}(t) \\
& -b_{i} w_{i}(t)+\sum_{j=1}^{n} c_{i j} \widetilde{P}_{j}\left(w_{j}(t)\right)+\sum_{j=1}^{n} d_{i j} \int_{0}^{+\infty} K_{i j}(u) \widetilde{Q}_{j}\left(w_{j}(t-u)\right) d u,
\end{aligned}
$$

where

$$
\widetilde{P}_{j}\left(w_{j}(t)\right)=P_{j}\left(y_{j}(t)\right)-P_{j}\left(x_{j}(t)\right), \quad \widetilde{Q}_{j}\left(w_{j}(t-u)\right)=Q_{j}\left(y_{j}(t-u)\right)-Q_{j}\left(x_{j}(t-u)\right), \quad j \in D .
$$

In the light of $\left(T_{2}\right)$, one can select a constant $\lambda \in\left(0, \frac{1}{2} \kappa\right]$ to agree with that

$$
E_{i}^{\lambda}<0, \quad 4 E_{i}^{\lambda} F_{i}^{\lambda}>\left(H_{i}^{\lambda}\right)^{2}, \quad i \in D
$$


where

$$
\left\{\begin{aligned}
E_{i}^{\lambda}= & \lambda \alpha_{i}^{2}+\alpha_{i} \gamma_{i}-a_{i} \alpha_{i}^{2}+\frac{1}{2} \alpha_{i}^{2} \sum_{j=1}^{n}\left(\left|c_{i j}\right| L_{j}^{P}+\left|d_{i j}\right| L_{j}^{Q} \int_{0}^{+\infty}\left|K_{i j}(u)\right| d u\right), \\
F_{i}^{\lambda}= & -b_{i} \alpha_{i} \gamma_{i}+\lambda \beta_{i}+\lambda \gamma_{i}^{2}+\frac{1}{2} \sum_{j=1}^{n}\left(\left|c_{i j}\right| L_{j}^{P}+\left|d_{i j}\right| L_{j}^{Q} \int_{0}^{+\infty}\left|K_{i j}(u)\right| d u\right)\left|\alpha_{i} \gamma_{i}\right| \\
& +\frac{1}{2} \sum_{j=1}^{n} \alpha_{j}^{2}\left(\left|c_{j i}\right| L_{i}^{P}+\left|d_{j i}\right| L_{i}^{Q} \int_{0}^{+\infty}\left|K_{j i}(u)\right| e^{2 \lambda u} d u\right) \\
& +\frac{1}{2} \sum_{j=1}^{n}\left(\left|c_{j i}\right| L_{i}^{P}+\left|d_{j i}\right| L_{i}^{Q} \int_{0}^{+\infty}\left|K_{j i}(u)\right| e^{2 \lambda u} d u\right)\left|\alpha_{j} \gamma_{j}\right|, \\
H_{i}^{\lambda}= & \beta_{i}+\gamma_{i}^{2}+2 \lambda \alpha_{i} \gamma_{i}-b_{i} \alpha_{i}^{2}-a_{i} \alpha_{i} \gamma_{i} .
\end{aligned}\right.
$$

Designate

$$
\begin{aligned}
\Pi(t)= & \frac{1}{2} \sum_{i=1}^{n} \beta_{i} w_{i}^{2}(t) e^{2 \lambda t}+\frac{1}{2} \sum_{i=1}^{n}\left(\alpha_{i} w_{i}^{\prime}(t)+\gamma_{i} w_{i}(t)\right)^{2} e^{2 \lambda t} \\
& +\frac{1}{2} \sum_{i=1}^{n} \sum_{j=1}^{n}\left(\alpha_{i}^{2}\left|d_{i j}\right|+\left|\alpha_{i} \gamma_{i}\right|\left|d_{i j}\right|\right) L_{j}^{Q} \int_{0}^{+\infty}\left|K_{i j}(u)\right| \int_{t-u}^{t} w_{j}^{2}(s) e^{2 \lambda(u+s)} d s d u .
\end{aligned}
$$

Differentiating $\Pi(t)$ on solutions along system (2.2) leads to

$$
\begin{aligned}
\Pi^{\prime}(t)= & 2 \lambda\left[\frac{1}{2} \sum_{i=1}^{n} \beta_{i} w_{i}^{2}(t) e^{2 \lambda t}+\frac{1}{2} \sum_{i=1}^{n}\left(\alpha_{i} w_{i}^{\prime}(t)+\gamma_{i} w_{i}(t)\right)^{2} e^{2 \lambda t}\right] \\
& +\sum_{i=1}^{n} \beta_{i} w_{i}(t) w_{i}^{\prime}(t) e^{2 \lambda t}+\sum_{i=1}^{n}\left(\alpha_{i} w_{i}^{\prime}(t)+\gamma_{i} w_{i}(t)\right)\left(\alpha_{i} w_{i}^{\prime \prime}(t)+\gamma_{i} w_{i}^{\prime}(t)\right) e^{2 \lambda t} \\
& +\frac{1}{2} \sum_{i=1}^{n} \sum_{j=1}^{n}\left(\alpha_{i}^{2}\left|d_{i j}\right|+\left|\alpha_{i} \gamma_{i}\right|\left|d_{i j}\right|\right) L_{j}^{Q} \int_{0}^{+\infty}\left|K_{i j}(u)\right|\left[w_{j}^{2}(t) e^{2 \lambda(u+t)}\right. \\
& \left.-w_{j}^{2}(t-u) e^{2 \lambda t}\right] d u \\
= & 2 \lambda\left[\frac{1}{2} \sum_{i=1}^{n} \beta_{i} w_{i}^{2}(t) e^{2 \lambda t}+\frac{1}{2} \sum_{i=1}^{n}\left(\alpha_{i} w_{i}^{\prime}(t)+\gamma_{i} w_{i}(t)\right)^{2} e^{2 \lambda t}\right] \\
& +\sum_{i=1}^{n}\left(\beta_{i}+\gamma_{i}^{2}\right) w_{i}(t) w_{i}^{\prime}(t) e^{2 \lambda t}+\sum_{i=1}^{n} \alpha_{i}\left(\alpha_{i} w_{i}^{\prime}(t)+\gamma_{i} w_{i}(t)\right) e^{2 \lambda t} \\
& \times\left[-a_{i} w_{i}^{\prime}(t)-b_{i} w_{i}(t)+\sum_{j=1}^{n} c_{i j} \widetilde{P}_{j}\left(w_{j}(t)\right)+\sum_{j=1}^{n} d_{i j} \int_{0}^{+\infty} K_{i j}(u) \widetilde{Q}_{j}\left(w_{j}(t-u)\right) d u\right] \\
& +\sum_{i=1}^{n} \alpha_{i} \gamma_{i}\left(w_{i}^{\prime}(t)\right)^{2} e^{2 \lambda t}+\frac{1}{2} \sum_{i=1}^{n} \sum_{j=1}^{n}\left(\alpha_{i}^{2}\left|d_{i j}\right|+\left|\alpha_{i} \gamma_{i}\right|\left|d_{i j}\right|\right) L_{j}^{Q} \\
& \times\left[\int_{0}^{+\infty}\left|K_{i j}(u)\right| e^{2 \lambda u} d u w_{j}^{2}(t) e^{2 \lambda t}-\int_{0}^{+\infty}\left|K_{i j}(u)\right| w_{j}^{2}(t-u) d u e^{2 \lambda t}\right] \\
\leq & e^{2 \lambda t}\left\{\sum_{i=1}^{n}\left(\beta_{i}+\gamma_{i}^{2}+2 \lambda \alpha_{i} \gamma_{i}-a_{i} \alpha_{i} \gamma_{i}-b_{i} \alpha_{i}^{2}\right) w_{i}(t) w_{i}^{\prime}(t)\right. \\
& \sum_{i=1}^{n}\left(\lambda \alpha_{i}^{2}+\alpha_{i} \gamma_{i}-a_{i} \alpha_{i}^{2}\right)\left(w_{i}^{\prime}(t)\right)^{2}-\sum_{i=1}^{n}\left(b_{i} \alpha_{i} \gamma_{i}-\lambda \beta_{i}-\lambda \gamma_{i}^{2}\right) w_{i}^{2}(t)
\end{aligned}
$$




$$
\begin{aligned}
& +\frac{1}{2} \sum_{i=1}^{n} \sum_{j=1}^{n}\left(\alpha_{i}^{2}\left|d_{i j}\right|+\left|\alpha_{i} \gamma_{i}\right|\left|d_{i j}\right|\right) L_{j}^{Q} \int_{0}^{+\infty}\left|K_{i j}(u)\right| e^{2 \lambda u} d u w_{j}^{2}(t) \\
& -\frac{1}{2} \sum_{i=1}^{n} \sum_{j=1}^{n}\left(\alpha_{i}^{2}\left|d_{i j}\right|+\left|\alpha_{i} \gamma_{i}\right|\left|d_{i j}\right|\right) L_{j}^{Q} \int_{0}^{+\infty}\left|K_{i j}(u)\right| w_{j}^{2}(t-u) d u \\
& +\sum_{i=1}^{n} \sum_{j=1}^{n}\left(\alpha_{i}^{2}\left|w_{i}^{\prime}(t)\right|+\left|\alpha_{i} \gamma_{i}\right|\left|w_{i}(t)\right|\right)\left|c_{i j}\right|\left|\widetilde{P}_{j}\left(w_{j}(t)\right)\right| \\
& \left.+\sum_{i=1}^{n} \sum_{j=1}^{n}\left(\alpha_{i}^{2}\left|w_{i}^{\prime}(t)\right|+\left|\alpha_{i} \gamma_{i}\right|\left|w_{i}(t)\right|\right)\left|d_{i j}\right| \int_{0}^{+\infty}\left|K_{i j}(u)\right|\left|\widetilde{Q}_{j}\left(w_{j}(t-u)\right)\right| d u\right\} \\
& =e^{2 \lambda t}\left\{\sum_{i=1}^{n}\left(\beta_{i}+\gamma_{i}^{2}+2 \lambda \alpha_{i} \gamma_{i}-a_{i} \alpha_{i} \gamma_{i}-b_{i} \alpha_{i}^{2}\right) w_{i}(t) w_{i}^{\prime}(t)\right. \\
& +\sum_{i=1}^{n}\left(\lambda \alpha_{i}^{2}+\alpha_{i} \gamma_{i}-a_{i} \alpha_{i}^{2}\right)\left(w_{i}^{\prime}(t)\right)^{2} \\
& +\sum_{i=1}^{n}\left[-b_{i} \alpha_{i} \gamma_{i}+\lambda \beta_{i}+\lambda \gamma_{i}^{2}+\frac{1}{2} \sum_{j=1}^{n}\left(\alpha_{j}^{2}\left|d_{j i}\right|+\left|\alpha_{j} \gamma_{j}\right|\left|d_{j i}\right|\right) L_{i}^{Q}\right. \\
& \left.+\int_{i=1}^{+\infty}\left|K_{j i}(u)\right| e^{2 \lambda u} d u\right] w_{i}^{2}(t) \\
& \left.+\int_{0}^{+\infty}\left|K_{i j}(u)\right|\left|\widetilde{Q}_{j}\left(w_{j}(t-u)\right)\right| d u\right\}, \quad \forall t \in[0,+\infty) . \\
& \left.\left.+\frac{1}{2} \sum_{i=1}^{n} \sum_{j=1}^{n}\left(\alpha_{i}^{2}\left|\alpha_{i}^{2}\right| d_{i j}|+| \alpha_{i} \gamma_{i}|| d_{i j} \mid\right) L_{j}^{G} \int_{0}^{+\infty}\left|\alpha_{i j}\right| \alpha_{i j}(u)\left|w_{j}^{\prime}(t)\right|+\left|\alpha_{i} \gamma_{i}\right|\left|w_{i}(t)\right|\right)\left|c_{i j}\right|\left|\widetilde{P}_{j}\left(w_{j}(t)\right)\right|\left|z_{i}(t)\right|\right)\left|d_{i j}\right| \\
& +u) d u
\end{aligned}
$$

With the help of (1.2) and the fact that $A B \leq \frac{1}{2}\left(A^{2}+B^{2}\right)(A, B \in \mathbb{R})$, one can see

$$
\begin{aligned}
& \sum_{i=1}^{n} \sum_{j=1}^{n}\left(\alpha_{i}^{2}\left|w_{i}^{\prime}(t)\right|+\left|\alpha_{i} \gamma_{i}\right|\left|w_{i}(t)\right|\right)\left|c_{i j}\right|\left|\widetilde{P}_{j}\left(w_{j}(t)\right)\right| \\
& \leq \frac{1}{2} \sum_{i=1}^{n} \sum_{j=1}^{n} \alpha_{i}^{2}\left|c_{i j}\right| L_{j}^{P}\left(\left(w_{i}^{\prime}(t)\right)^{2}+w_{j}^{2}(t)\right) \\
& \quad+\frac{1}{2} \sum_{i=1}^{n} \sum_{j=1}^{n}\left|\alpha_{i} \gamma_{i}\right|\left|c_{i j}\right| L_{j}^{P}\left(w_{i}^{2}(t)+w_{j}^{2}(t)\right) \\
& =\frac{1}{2} \sum_{i=1}^{n} \sum_{j=1}^{n} \alpha_{i}^{2}\left|c_{i j}\right| L_{j}^{P}\left(w_{i}^{\prime}(t)\right)^{2}+\frac{1}{2} \sum_{i=1}^{n} \sum_{j=1}^{n}\left(\left|\alpha_{i} \gamma_{i}\right|\left|c_{i j}\right| L_{j}^{P}+\alpha_{j}^{2}\left|c_{j i}\right| L_{i}^{P}\right. \\
& \left.\quad+\left|\alpha_{j} \gamma_{j}\right|\left|c_{j i}\right| L_{i}^{P}\right) w_{i}^{2}(t)
\end{aligned}
$$


and

$$
\begin{aligned}
\sum_{i=1}^{n} & \sum_{j=1}^{n}\left(\alpha_{i}^{2}\left|w_{i}^{\prime}(t)\right|+\left|\alpha_{i} \gamma_{i}\right|\left|w_{i}(t)\right|\right)\left|d_{i j}\right| \int_{0}^{+\infty}\left|K_{i j}(u)\right|\left|\widetilde{Q}_{j}\left(z_{j}(t-u)\right)\right| d u \\
\leq & \frac{1}{2} \sum_{i=1}^{n} \sum_{j=1}^{n} \alpha_{i}^{2}\left|d_{i j}\right| L_{j}^{Q} \int_{0}^{+\infty}\left|K_{i j}(u)\right|\left(\left(w_{i}^{\prime}(t)\right)^{2}+w_{j}^{2}(t-u)\right) d u \\
& +\frac{1}{2} \sum_{i=1}^{n} \sum_{j=1}^{n}\left|\alpha_{i} \gamma_{i}\right|\left|d_{i j}\right| L_{j}^{Q} \int_{0}^{+\infty}\left|K_{i j}(u)\right|\left(w_{i}^{2}(t)+z_{j}^{2}(t-u)\right) d u \\
= & \frac{1}{2} \sum_{i=1}^{n} \sum_{j=1}^{n} \alpha_{i}^{2}\left|d_{i j}\right| L_{j}^{Q} \int_{0}^{+\infty}\left|K_{i j}(u)\right| d u\left(w_{i}^{\prime}(t)\right)^{2} \\
& +\frac{1}{2} \sum_{i=1}^{n} \sum_{j=1}^{n}\left|\alpha_{i} \gamma_{i}\right|\left|d_{i j}\right| L_{j}^{Q} \int_{0}^{+\infty}\left|K_{i j}(u)\right| d u w_{i}^{2}(t) \\
& +\frac{1}{2} \sum_{i=1}^{n} \sum_{j=1}^{n}\left(\alpha_{i}^{2}\left|d_{i j}\right| L_{j}^{Q}+\left|\alpha_{i} \gamma_{i}\right|\left|d_{i j}\right| L_{j}^{Q}\right) \int_{0}^{+\infty}\left|K_{i j}(u)\right| w_{j}^{2}(t-u) d u
\end{aligned}
$$

which, along with (2.3) and (2.4), results in

$$
\begin{aligned}
& \Pi^{\prime}(t) \leq e^{2 \lambda t}\left\{\sum_{i=1}^{n}\left(\beta_{i}+\gamma_{i}^{2}+2 \lambda \alpha_{i} \gamma_{i}-a_{i} \alpha_{i} \gamma_{i}-b_{i} \alpha_{i}^{2}\right) w_{i}(t) w_{i}^{\prime}(t)\right. \\
& +\sum_{i=1}^{n}\left[\lambda \alpha_{i}^{2}+\alpha_{i} \gamma_{i}-a_{i} \alpha_{i}^{2}\right. \\
& \left.+\frac{1}{2} \alpha_{i}^{2} \sum_{j=1}^{n}\left(\left|c_{i j}\right| L_{j}^{P}+\left|d_{i j}\right| L_{j}^{Q} \int_{0}^{+\infty}\left|K_{i j}(u)\right| d u\right)\right]\left(w_{i}^{\prime}(t)\right)^{2} \\
& +\sum_{i=1}^{n}\left[-b_{i} \alpha_{i} \gamma_{i}+\lambda \beta_{i}+\lambda \gamma_{i}^{2}+\frac{1}{2} \sum_{j=1}^{n}\left(\left|c_{i j}\right| L_{j}^{P}+\left|d_{i j}\right| L_{j}^{Q} \int_{0}^{+\infty}\left|K_{i j}(u)\right| d u\right)\left|\alpha_{i} \gamma_{i}\right|\right. \\
& +\frac{1}{2} \sum_{j=1}^{n} \alpha_{j}^{2}\left(\left|c_{j i}\right| L_{i}^{P}+\left|d_{j i}\right| L_{i}^{Q} \int_{0}^{+\infty}\left|K_{j i}(u)\right| e^{2 \lambda u} d u\right) \\
& \left.\left.+\frac{1}{2} \sum_{j=1}^{n}\left(\left|c_{j i}\right| L_{i}^{P}+\left|d_{j i}\right| L_{i}^{Q} \int_{0}^{+\infty}\left|K_{j i}(u)\right| e^{2 \lambda u} d u\right)\left|\alpha_{j} \gamma_{j}\right|\right] w_{i}^{2}(t)\right\} \\
& =e^{2 \lambda t}\left\{\sum_{i=1}^{n}\left(E_{i}^{\lambda}\left(z_{i}^{\prime}(t)\right)^{2}+F_{i}^{\lambda} w_{i}^{2}(t)+H_{i}^{\lambda} w_{i}(t) w_{i}^{\prime}(t)\right)\right\} \\
& =e^{2 \lambda t}\left\{\sum_{i=1}^{n} E_{i}^{\lambda}\left(w_{i}^{\prime}(t)+\frac{H_{i}^{\lambda}}{2 E_{i}^{\lambda}} w_{i}(t)\right)^{2}+\sum_{i=1}^{n}\left(F_{i}^{\lambda}-\frac{\left(H_{i}^{\lambda}\right)^{2}}{4 E_{i}^{\lambda}}\right) w_{i}^{2}(t)\right\} \\
& \leq 0, \quad \forall t \in[0,+\infty) \text {. }
\end{aligned}
$$


This indicates that $\Pi(t) \leq \Pi(0)$ on $[0,+\infty)$ and

$$
\frac{1}{2} \sum_{i=1}^{n} \beta_{i} z_{i}^{2}(t) e^{2 \lambda t}+\frac{1}{2} \sum_{i=1}^{n}\left(\alpha_{i} z_{i}^{\prime}(t)+\gamma_{i} z_{i}(t)\right)^{2} e^{2 \lambda t} \leq U(0), \quad t \in[0,+\infty) .
$$

Moreover, by using the Cauchy-Schwarz inequality,

$$
\left(\alpha_{i} w_{i}^{\prime}(t) e^{\lambda t}+\gamma_{i} w_{i}(t) e^{\lambda t}\right)^{2}=\left(\alpha_{i} w_{i}^{\prime}(t)+\gamma_{i} w_{i}(t)\right)^{2} e^{2 \lambda t}
$$

and

$$
\alpha_{i}\left|w_{i}^{\prime}(t)\right| e^{\lambda t} \leq\left|\alpha_{i} w_{i}^{\prime}(t) e^{\lambda t}+\gamma_{i} w_{i}(t) e^{\lambda t}\right|+\left|\gamma_{i} w_{i}(t) e^{\lambda t}\right|
$$

imply that one can take a positive constant $\Lambda_{\varphi, \psi}>0$ satisfying

$$
\left|w_{i}^{\prime}(t)\right| \leq \Lambda_{\varphi, \psi} e^{-\lambda t}, \quad\left|w_{i}(t)\right| \leq \Lambda_{\varphi, \psi} e^{-\lambda t}, \quad \forall t \geq 0, i \in D,
$$

which finishes the proof of Theorem 2.1.

Corollary 2.1 Under (1.2), define $J_{i}(t) \equiv J$ as a constant, and

$$
\beta_{i}=\alpha_{i}^{2} b_{i}+a_{i} \gamma_{i} \alpha_{i}-\gamma_{i}^{2}>0, \quad E_{i}<0, \quad F_{i}<0, \quad i \in D .
$$

Then the equilibrium point in (1.1) is globally exponentially stable.

Proof With the aid of the proof of Corollary 2 in [36], one can reveal that (1.1) has exactly one equilibrium $y^{*}$. Then Remark 2.1 and Theorem 2.1 give that $y^{*}$ is globally exponentially stable. This ends the proof.

Remark 2.2 Since the authors in [36] have not considered the exponential stability on inertial neural networks by using non-reduced order method, our results in Theorem 2.1 and Corollary 2.1 not only improve the main conclusions of [36], but also generalize them. Furthermore, using an argument similar to the one adopted in Theorem 2.1, it is not difficult to obtain the global exponential synchronization of networks (1.1) subject to its driving system.

\section{Some simulations}

In this section, we give some numeric simulation results to verify our theoretical results. We choose the following models:

$$
\left\{\begin{aligned}
x_{1}^{\prime \prime}(t)= & -3.81 x_{1}^{\prime}(t)-8.21 x_{1}(t)+1.21 P_{1}\left(x_{1}(t)\right)+1.51 P_{2}\left(x_{2}(t)\right) \\
& -0.81 \int_{0}^{+\infty}(\sin 2 u) e^{-u} Q_{1}\left(x_{1}(t-u)\right) d u \\
& +1.91 \int_{0}^{+\infty}(\sin 3 u) e^{-u} Q_{2}\left(x_{2}(t-u)\right) d u+10 \sin t \\
x_{2}^{\prime \prime}(t)= & -4.71 x_{2}^{\prime}(t)-10.91 x_{2}(t)-0.91 P_{1}\left(x_{1}(t)\right)-1.71 P_{2}\left(x_{2}(t)\right) \\
& -2.51 \int_{0}^{+\infty}(\sin 4 u) e^{-u} Q_{1}\left(x_{1}(t-u)\right) d u \\
& +2.11 \int_{0}^{+\infty}(\sin 5 u) e^{-u} Q_{2}\left(x_{2}(t-u)\right) d u+10 \cos t, \\
P_{i}(u)= & Q_{i}(u)=0.25(|u+1|-|u-1|), \quad i=1,2,
\end{aligned}\right.
$$




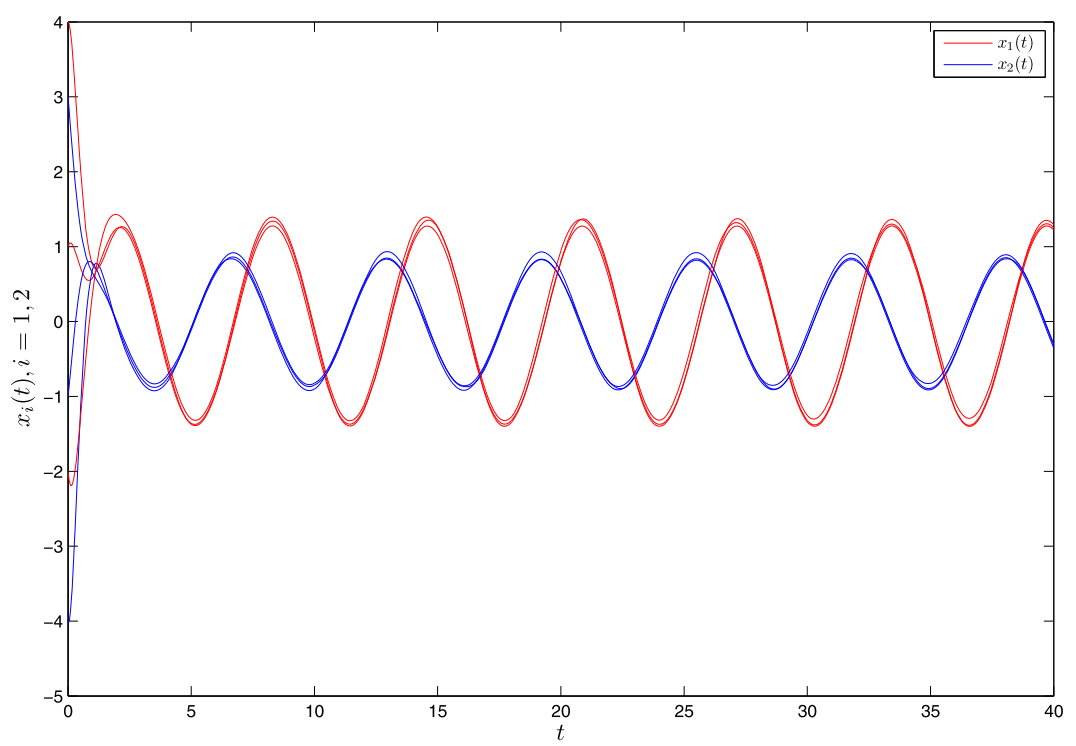

Figure 1 Numerical solutions $x(t)$ on system (3.1). Numerical solutions $x(t)$ to example (3.1) with initial values: $(\sin t+1,-\cos t-3, \cos t, \sin t),(2 \cos t+2,3 \sin t-1,-2 \sin t, 3 \cos t),(-3 \sin t-2,-4 \sin t+3,-3 \cos t,-4 \cos t)$

and

$$
\left\{\begin{aligned}
x_{1}^{\prime \prime}(t)= & -3.81 x_{1}^{\prime}(t)-8.21 x_{1}(t)+1.21 P_{1}\left(x_{1}(t)\right)+1.51 P_{2}\left(x_{2}(t)\right) \\
& -0.81 \int_{0}^{+\infty}(\sin 2 u) e^{-u} Q_{1}\left(x_{1}(t-u)\right) d u \\
& +1.91 \int_{0}^{+\infty}(\sin 3 u) e^{-u} Q_{2}\left(x_{2}(t-u)\right) d u+20, \\
x_{2}^{\prime \prime}(t)= & -4.71 x_{2}^{\prime}(t)-10.91 x_{2}(t)-0.91 P_{1}\left(x_{1}(t)\right)-1.71 P_{2}\left(x_{2}(t)\right) \\
& -2.51 \int_{0}^{+\infty}(\sin 4 u) e^{-u} Q_{1}\left(x_{1}(t-u)\right) d u \\
& +2.11 \int_{0}^{+\infty}(\sin 5 u) e^{-u} Q_{2}\left(x_{2}(t-u)\right) d u+20, \\
P_{i}(u)= & Q_{i}(u)=\frac{1}{4 \pi} \arctan u, \quad i=1,2 .
\end{aligned}\right.
$$

It is easy to check that (3.1) and (3.2) satisfy all the conditions made in Theorem 2.1 and Corollary 2.1, respectively. Consequently, (3.1) and (3.2) are globally exponentially stable. The numeric simulations in Figs. 1-4 support the theoretical results in Sect. 2.

Remark 3.1 Because the exponential stability of unbounded distributed delayed inertial neural networks has never been touched by the aid of the non-reduced order method, it is clear to find that all results in the references [15-37,39-78] cannot be straightly employed to reveal the exponential convergence on the solutions and their derivative for networks (3.1) and (3.2).

\section{Conclusions}

In this article, without utilizing the reduced order technique, the global exponential stability of unbounded continuously distributed delayed inertial neural networks has been considered. By combining Lyapunov function way with differential inequality analysis, some sufficient assertions have been gained to evidence the global exponential convergence on 


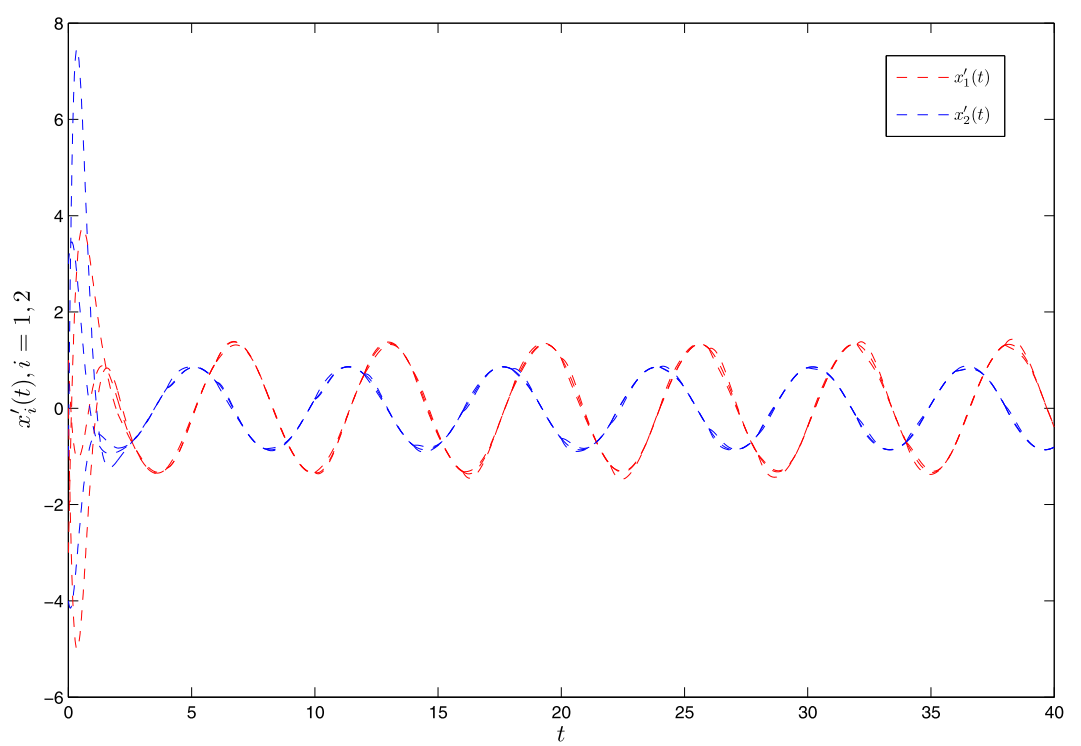

Figure 2 Numerical solutions $x^{\prime}(t)$ on system (3.1). Numerical solutions $x^{\prime}(t)$ to example (3.1) with initial values:

$(\sin t+1,-\cos t-3, \cos t, \sin t),(2 \cos t+2,3 \sin t-1,-2 \sin t, 3 \cos t),(-3 \sin t-2,-4 \sin t+3,-3 \cos t,-4 \cos t)$

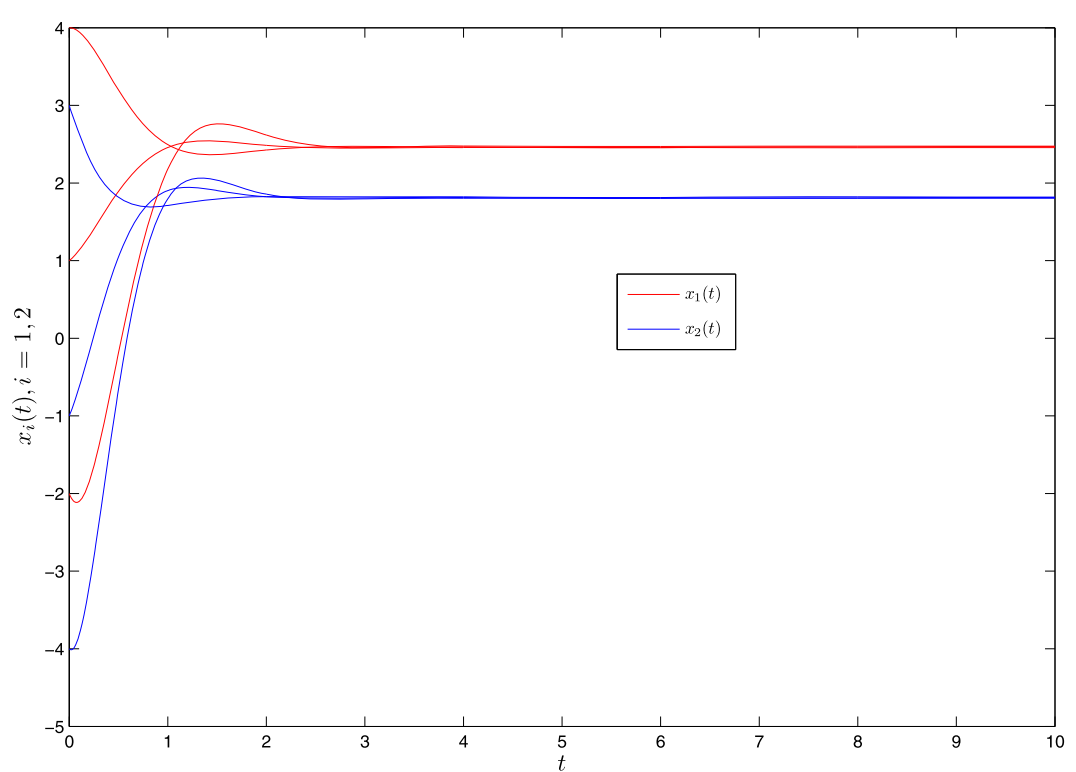

Figure 3 Numerical solutions $x(t)$ on system (3.2). Numerical solutions $x(t)$ to example (3.2) with initial values: $(\sin t+1,-\cos t-3, \cos t, \sin t),(2 \cos t+2,3 \sin t-1,-2 \sin t, 3 \cos t),(-3 \sin t-2,-4 \sin t+3,-3 \cos t,-4 \cos t)$

all solution and their derivatives in the addressed networks. It should be pointed out that our assumptions are easily checked in practice by simple inequality technique, and the approach adopted in this paper provides a possible way to investigate the dynamic topic on other unbounded continuously distributed delayed inertial neural networks. We would like to extend our approach to study the periodicity and dissipativity for unbounded distributed delayed inertial neural network models. 


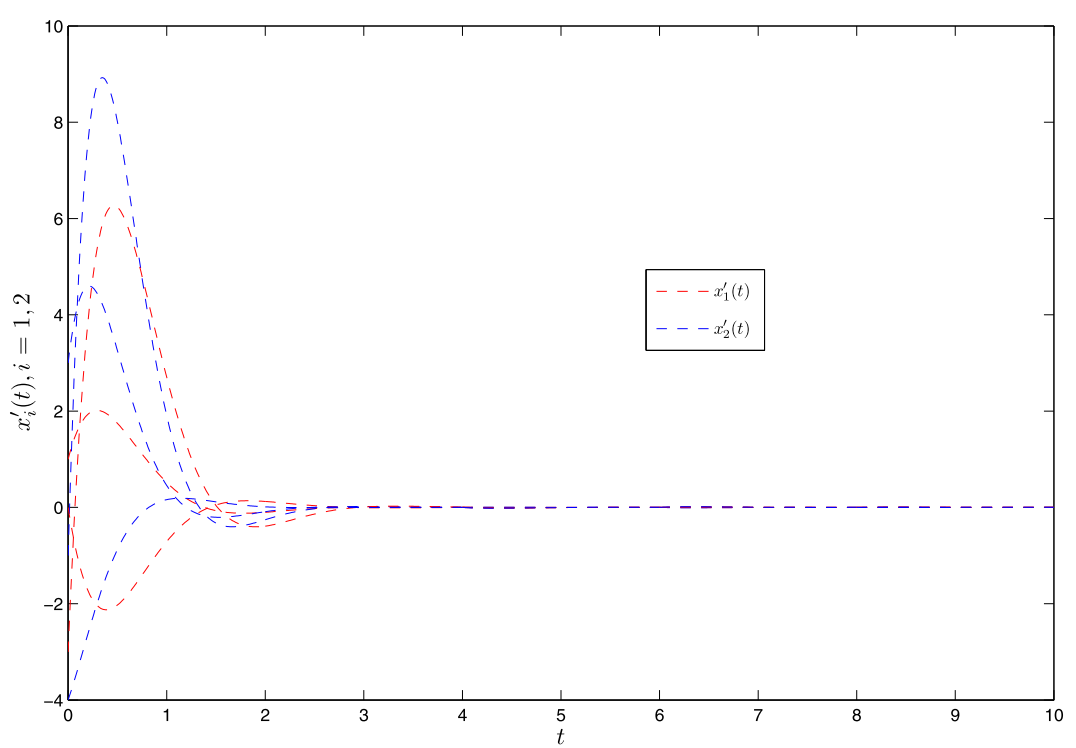

Figure 4 Numerical solutions $x^{\prime}(t)$ on system (3.2). Numerical solutions $x^{\prime}(t)$ to example (3.2) with initial values:

$(\sin t+1,-\cos t-3, \cos t, \sin t),(2 \cos t+2,3 \sin t-1,-2 \sin t, 3 \cos t),(-3 \sin t-2,-4 \sin t+3,-3 \cos t,-4 \cos t)$

\section{Acknowledgements}

We would like to thank the anonymous referees and the editor for considering our revised paper.

\section{Funding}

This work was supported by the National Natural Science Foundation of China (Nos. 11971076, 11771059, 51839002).

\section{Availability of data and materials}

Data sharing not applicable to this article as no datasets were generated or analysed during the current study.

\section{Competing interests}

The authors declare that they have no competing interests.

\section{Authors' contributions}

$\mathrm{CH}$ and JZ contributed equally to this revised work. All authors read and approved the final manuscript.

\section{Publisher's Note}

Springer Nature remains neutral with regard to jurisdictional claims in published maps and institutional affiliations.

\section{Received: 12 December 2019 Accepted: 28 February 2020 Published online: 16 March 2020}

\section{References}

1. Jiang, A.: Exponential convergence for shunting inhibitory cellular neural networks with oscillating coefficients inleakage terms. Neurocomputing 165, 159-162 (2015)

2. Cao, J., Manivannan, R., Chong, K.T., Lv, X.: Extended dissipativity performance of high-speed train including actuator faults and probabilistic time-delays under resilient reliable control. IEEE Trans. Syst. Man Cybern. Syst. (2019). https://doi.org/10.1109/TSMC.2019.2930997

3. Zhao, C., Wang, Z.: Exponential convergence of a SICNN with leakage delays and continuously distributed delays of neutral type. Neural Process. Lett. 41, 239-247 (2015)

4. Cao, J., Stamov, G. Stamova, l., Simeonov, S.: Almost periodicity in impulsive fractional-order reaction-diffusion neural networks with time-varying delays. IEEE Trans. Cybern. 2020, 2967625 (2020) https://doi.org/10.1109/TCYB.2020.2967625

5. Zhang, H.: Existence and stability of almost periodic solutions for CNNs with continuously distributed leakage delays. Neural Comput. Appl. 24, 1135-1146 (2014)

6. Huang, C., Long, X., Cao, J.: Stability of anti-periodic recurrent neural networks with multi-proportional delays. Math Methods Appl. Sci. 2020, 6350 (2020). https://doi.org/10.1002/mma.6350

7. Chen, D., Zhang, W., Cao, J., Huang, C.: Fixed time synchronization of delayed quaternion-valued memristor-based neural Networks. Adv. Differ. Equ. 2020, 92 (2020). https://doi.org/10.1186/s13662-020-02560-w

8. Xu, Y.: Exponential stability of weighted pseudo almost periodic solutions for HCNNs with mixed delays. Neural Process. Lett. 46, 507-519 (2017) 
9. Huang, C., Liu, B., Tian, X., et al.: Global convergence on asymptotically almost periodic SICNNs with nonlinear decay functions. Neural Process. Lett. 49, 625-641 (2019)

10. Zhou, Y., Wan, X., Huang, C., Yang, X.: Finite-time stochastic synchronization of dynamic networks with nonlinear coupling strength via quantized intermittent control. Appl. Math. Comput. 376, Article 125157 (2020). https://doi.org/10.1016/j.amc.2020.125157

11. Zhou, Q.: Weighted pseudo anti-periodic solutions for cellular neural networks with mixed delays. Asian J. Control 19(4), 1557-1563 (2017)

12. Zhou, Q., Shao, J.: Weighted pseudo anti-periodic SICNNs with mixed delays. Neural Comput. Appl. 29, 865-872 (2018)

13. Babcock, K., Westervelt, R.: Stability and dynamics of simple electronic neural networks with added inertia. Physica D 23, 464-469 (1986)

14. Babcock, K., Westervelt, R.: Dynamics of simple electronic neural networks. Physica D 28, 305-316 (1987)

15. Ke, Y., Miao, C.: Stability analysis of inertial Cohen-Grossberg-type neural networks with time delays. Neurocomputing 117, 196-205 (2013)

16. Yu, S., Zhang, Z., Quan, Z.: New global exponential stability conditions for inertial Cohen-Grossberg neural networks with time delays. Neurocomputing 151, 1446-1454 (2015)

17. Zhang, Z., Quan, Z.: Global exponential stability via inequality technique for inertial BAM neural networks with time delays. Neurocomputing 151, 1316-1326 (2015)

18. Wang, J., Tian, L.: Global Lagrange stability for inertial neural networks with mixed time varying delays. Neurocomputing 235, 140-146 (2017)

19. Li, J., Ying, J., Xie, D.: On the analysis and application of an ion size-modified Poisson-Boltzmann equation. Nonlinear Anal., Real World Appl. 47, 188-203 (2019)

20. Wang, W., Chen, Y., Fang, H.: On the variable two-step IMEX BDF method for parabolic integro-differential equations with nonsmooth initial data arising in finance. SIAM J. Numer. Anal. 57(3), 1289-1317 (2019)

21. Tang, W., Sun, Y., Zhang, J.: High order symplectic integrators based on continuous-stage Runge-Kutta-Nystrom methods. Appl. Math. Comput. 361, 670-679 (2019)

22. Tian, Z., Liu, Y., Zhang, Y., Liu, Z., Tian, M.: The general inner-outer iteration method based on regular splittings for the PageRank problem. Appl. Math. Comput. 356, 479-501 (2019)

23. Jiang, Y., Xu, X.: A monotone finite volume method for time fractional Fokker-Planck equations. Sci. China Math. 62(4), 783-794 (2019)

24. Chen, H., Xu, D., Zhou, J.: A second-order accurate numerical method with graded meshes for an evolution equation with a weakly singular kernel. J. Comput. Appl. Math. 356, 152-163 (2019)

25. Yu, B., Fan, H.Y., Chu, E.K.: Large-scale algebraic Riccati equations with high-rank constant terms. J. Comput. Appl. Math. 361, 130-143 (2019)

26. Tang, W., Zhang, J.: Symmetric integrators based on continuous-stage Runge-Kutta-Nystrom methods for reversible systems. Appl. Math. Comput. 361, 1-12 (2019)

27. Liu, F., Feng, L., Anh, V., Li, J.: Unstructured-mesh Galerkin finite element method for the two-dimensional multi-term time-space fractional Bloch-Torrey equations on irregular convex domains. Comput. Math. Appl. 78(5), 1637-1650 (2019)

28. Zhou, S., Jiang, Y.: Finite volume methods for N-dimensional time fractional Fokker-Planck equations. Bull. Malays. Math. Sci. Soc. 42(6), 3167-3186 (2019)

29. Duan, L., Huang, C.: Existence and global attractivity of almost periodic solutions for a delayed differential neoclassical growth model. Math. Methods Appl. Sci. 40(3), 814-822 (2017)

30. Huang, C., Yang, L., Liu, B.: New results on periodicity of non-autonomous inertial neural networks involving non-reduced order method. Neural Process. Lett. 50, 595-606 (2019)

31. Huang, C.: Exponential stability of inertial neural networks involving proportional delays and non-reduced order method. J. Exp. Theor. Artif. Intell. 32, 133-146 (2020)

32. Huang, C., Wen, S., Huang, L.: Dynamics of anti-periodic solutions on shunting inhibitory cellular neural networks with multi-proportional delays. Neurocomputing 357(10), 47-52 (2019)

33. Huang, C., Yang, Z., Yi, T., Zou, X.: On the basins of attraction for a class of delay differential equations with non-monotone bistable nonlinearities. J. Differ. Equ. 256, 2101-2114 (2014)

34. Huang, C., Zhang, H.: Periodicity of non-autonomous inertial neural networks involving proportional delays and non-reduced order method. Int. J. Biomath. 12(2), Article ID 1950016 (2019)

35. Huang, C., Zhang, H., Huang, L.: Almost periodicity analysis for a delayed Nicholson's blowflies model with nonlinear density-dependent mortality term. Commun. Pure Appl. Anal. 18(6), 3337-3349 (2019)

36. Li, X., Li, X., Hu, C.: Some new results on stability and synchronization for delayed inertial neural networks based on non-reduced order method. Neural Netw. 96, 91-100 (2017)

37. Huang, C., Liu, B.: New studies on dynamic analysis of inertial neural networks involving non-reduced order method. Neurocomputing 325(24), 283-287 (2019)

38. Hino, Y., Murakami, S., Nai, T.: Functional Differential Equations with Infinite Delay. Lecture in Mathematics, vol. 1473. Springer, Berlin (1991)

39. Xu, Y.: Convergence on non-autonomous inertial neural networks with unbounded distributed delays. J. Exp. Theor. Artif. Intell. (2019). https://doi.org/10.1080/0952813X.2019.1652941

40. Cai, Z., Huang, J., Huang, L.: Periodic orbit analysis for the delayed Filippov system. Proc. Am. Math. Soc. 146, 4667-4682 (2018)

41. Li, Y., Vuorinen, M., Zhou, Q:: Apollonian metric, uniformity and Gromov hyperbolicity. Complex Var. Elliptic Equ. (2019). https://doi.org/10.1080/17476933.2019.1579203

42. Huang, C., Qiao, Y., Huang, L., Agarwal, R.P.: Dynamical behaviors of a food-chain model with stage structure and time delays. Adv. Differ. Equ. 2018, 186 (2018). https://doi.org/10.1186/s13662-018-1589-8

43. Li, X., Liu, Z., Li, J.: Existence and controllability for nonlinear fractional control systems with damping in Hilbert spaces. Acta Math. Sin. Engl. Ser. 39(1), 229-242 (2019)

44. Zhu, K., Xie, Y., Zhou, F.: Pullback attractors for a damped semilinear wave equation with delays. Acta Math. Sin. Engl. Ser. 34(7), 1131-1150 (2018) 
45. Zhao, J., Liu, J., Fang, L.: Anti-periodic boundary value problems of second-order functional differential equations. Bull. Malays. Math. Sci. Soc. 37(2), 311-320 (2014)

46. Long, X., Gong, S.: New results on stability of Nicholson's blowflies equation with multiple pairs of time-varying delays. Appl. Math. Lett. 100, 106027 (2020). https://doi.org/10.1016/j.aml.2019.106027

47. Duan, L., Fang, X., Huang, C.: Global exponential convergence in a delayed almost periodic Nicholson's blowflies model with discontinuous harvesting. Math. Methods Appl. Sci. 41(5), 1954-1965 (2018)

48. Huang, C., Zhang, H., Cao, J., Hu, H.: Stability and Hopf bifurcation of a delayed prey-predator model with disease in the predator. Int. J. Bifurc. Chaos 29(7), Article ID 1950091 (2019)

49. Huang, C., Yang, X., Cao, J.: Stability analysis of Nicholson's blowflies equation with two different delays. Math. Comput. Simul. 171, 201-206 (2020)

50. Tan, Y., Huang, C., Sun, B., Wang, T.: Dynamics of a class of delayed reaction-diffusion systems with Neumann boundary condition. J. Math. Anal. Appl. 458(2), 1115-1130 (2018)

51. Li, L., Wang, W., Huang, L., Wu, J.: Some weak flocking models and its application to target tracking. J. Math. Anal. Appl. 480(2), Article 123404 (2019). https://doi.org/10.1016/j.jmaa.2019.123404

52. Chen, T., Huang, L., Yu, P., Huang, W.: Bifurcation of limit cycles at infinity in piecewise polynomial systems. Nonlinear Anal., Real World Appl. 41, 82-106 (2018)

53. Hu, H., Zou, X.: Existence of an extinction wave in the Fisher equation with a shifting habitat. Proc. Am. Math. Soc. 145(11), 4763-4771 (2017)

54. Wang, J., Huang, C., Huang, L.: Discontinuity-induced limit cycles in a general planar piecewise linear system of saddle-focus type. Nonlinear Anal. Hybrid Syst. 33, 162-178 (2019)

55. Wang, J., Chen, X., Huang, L.: The number and stability of limit cycles for planar piecewise linear systems of node-saddle type. J. Math. Anal. Appl. 469(1), 405-427 (2019)

56. Yang, X., Wen, S., Liu, Z., Li, C., Huang, C.: Dynamic properties of foreign exchange complex network. Mathematics 7(9), 832 (2019). https://doi.org/10.3390/math7090832

57. Iswarya, M., Raja, R., Rajchakit, G., Cao, J., Alzabut, J., Huang, C.: Existence, uniqueness and exponential stability of periodic solution for discrete-time delayed BAM neural networks based on coincidence degree theory and graph theoretic method. Mathematics (2019). https://doi.org/10.3390/math7111055

58. Zhang, H.: Global large smooth solutions for 3-d hall-magnetohydrodynamics. Discrete Contin. Dyn. Syst. 39(11), 6669-6682 (2019)

59. Li, W., Huang, L., Ji, J.: Periodic solution and its stability of a delayed Beddington-DeAngelis type predator-prey system with discontinuous control strategy. Math. Methods Appl. Sci. 42(13), 4498-4515 (2019)

60. Wang, F., Yao, Z: Approximate controllability of fractional neutral differential systems with bounded delay. Fixed Point Theory 17(2), 495-507 (2016)

61. Qian, C., Hu, Y.: Novel stability criteria on nonlinear density-dependent mortality Nicholson's blowflies systems in asymptotically almost periodic environments. J. Inequal. Appl. 2020, 13 (2020). https://doi.org/10.1186/s13660-019-2275-4

62. Huang, C., Long, X., Huang, L., Fu, S.: Stability of almost periodic Nicholson's blowflies model involving patch structure and mortality terms. Can. Math. Bull. (2019). https://doi.org/10.4153/S0008439519000511

63. Hu, H., Yuan, X., Huang, L., Huang, C.: Global dynamics of an SIRS model with demographics and transfer from infectious to susceptible on heterogeneous networks. Math. Biosci. Eng. 16(5), 5729-5749 (2019)

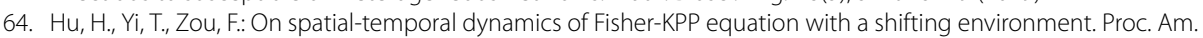
Math. Soc. 148, 213-221 (2020)

65. Zhang, J., Lu, C., Li, X., Kim, H.-J., Wang, J.: A full convolutional network based on DenseNet for remote sensing scene classification. Math. Biosci. Eng. 16(5), 3345-3367 (2019)

66. Huang, Y., Chen, X., Zhu, H., Huang, C., Tian, Z.: The heterogeneous effects of FDI and foreign trade on $\mathrm{CO} 2$ emissions: evidence from China. Math. Probl. Eng. 2019, Article ID 9612492 (2019). https://doi.org/10.1155/2019/9612492

67. Li, X., Yang, X., Huang, T.: Persistence of delayed cooperative models: impulsive control method. Appl. Math. Comput. $342,130-146(2019)$

68. Tan, Y., Liu, L.: Boundedness of Toeplitz operators related to singular integral operators. Izv. Math. 82(6), 1225-1238 (2018)

69. $\mathrm{Hu}, \mathrm{H} ., \mathrm{Liu}, \mathrm{L}$.: Weighted inequalities for a general commutator associated to a singular integral operator satisfying a variant of Hormander's condition. Math. Notes 101(5), 830-840 (2017)

70. Huang, C., Cao, J., Wen, F., Yang, X.: Stability analysis of SIR model with distributed delay on complex networks. PLoS ONE 11(8), Article ID e0158813 (2016)

71. Cao, Y., Samidurai, R., Sriraman, R.: Stability and dissipativity analysis for neutral type stochastic Markovian jump static neural networks with time delays. J. Artif. Intell. Softw. Comput. Res. 9(3), 189-204 (2019)

72. Cao, Y., Sriraman, R., Shyamsundarraj, N., Samidurai, R.: Robust stability of uncertain stochastic complex-valued neural networks with additive time-varying delays. Math. Comput. Simul. 171(3), 207-220 (2020)

73. Cao, Y., Sriraman, R., Samidurai, R.: Stability and stabilization analysis of nonlinear time-delay systems with randomly occurring controller gain fluctuation. Math. Comput. Simul. 171(3), 36-51 (2020)

74. Cao, Q., Wang, G., Qian, C.: New results on global exponential stability for a periodic Nicholson's blowflies model involving time-varying delays. Adv. Differ. Equ. 2020, 43 (2020). https://doi.org/10.1186/s13662-020-2495-4

75. Yang, D., Li, X., Qiu, J.: Output tracking control of delayed switched systems via state-dependent switching and dynamic output feedback. Nonlinear Anal. Hybrid Syst. 32, 294-305 (2019)

76. Yang, X., Li, X., Xi, Q., Duan, P.: Review of stability and stabilization for impulsive delayed systems. Math. Biosci. Eng. 15(6), 1495 (2018)

77. Cai, Z., Huang, L., Wang, Z.: Mono/multi-periodicity generated by impulses control in time-delayed memristor-based neural networks. Nonlinear Anal. Hybrid Syst. 36, 100861 (2020). https://doi.org/10.1016/j.nahs.2020.100861

78. Xu, Y., Cao, Q., Guo, X.: Stability on a patch structure Nicholson's blowflies system involving distinctive delays. Appl. Math. Lett. (2020). https://doi.org/10.1016/j.aml.2020.106340 